\title{
Symptomatic and Asymptomatic Colon Cancer Recurrence: A Multicenter Cohort Study
}

Laura A. M. Duineveld, $M D^{1}$

Kristel M. van Asselt, $M D, P b D^{1}$

Willem A. Bemelman, $M D, P b D^{2}$

Anke B. Smits, MD, PhD

Pieter J. Tanis, MD, $P b D^{2}$

Henk C. P. M. van Weert, MD, $P b D^{1}$

Jan Wind, $M D, P b D^{1}$

'Department of Primary Care, Academic Medical Centre, Amsterdam, the Netherlands

${ }^{2}$ Department of Surgery, Academic Medical Centre, Amsterdam, the Netherlands

${ }^{3}$ Department of Surgery, St Antonius Hospital, Nieuwegein, the Netherlands

Conflicts of interest: authors report none.

\section{CORRESPONDING AUTHOR}

Laura Duineveld

Department of Primary Care

Academic Medical Centre, University of Amsterdam

Post-box 22660, 1100 DD Amsterdam

1.a.duineveld@amc.uva.nl

\begin{abstract}
PURPOSE Follow-up after colorectal cancer treatment with curative intent aims to detect recurrences and metachronous tumors in a timely manner. The objective of this study is to assess how recurrent disease presents and is diagnosed within scheduled follow-up according to the national guideline for the Netherlands.
\end{abstract}

METHODS In a retrospective study of consecutive patients with colorectal cancer who were treated in 2 hospitals in the Netherlands, we identified patients with colon cancer who underwent surgery with curative intent between January 2007 and December 2012. Patients who developed recurrent disease were included for further analyses.

RESULTS From a total of 446 patients who were been treated for colon carcinoma with curative intent, 74 developed recurrent disease (17\%). In 43 of those patients (58\%), recurrent disease was detected during a scheduled follow-up visit, with 41 (95\%) being asymptomatic. Tumor marker testing, imaging, and colonoscopy identified all of these recurrences. In the remaining 31 patients with recurrent disease $(42 \%)$, recurrence was found during non-scheduled interval visits; $26(84 \%)$ of these patients were symptomatic. The most prevalent symptoms were abdominal pain, altered defecation, and weight loss. Patients with asymptomatic recurrences had a significantly higher overall survival compared with patients with symptomatic recurrences.

CONCLUSIONS In this cohort, $42 \%$ of the recurrences after initial curative treatment for colon cancer were found during non-scheduled interval visits, mainly based on symptoms. Primary care physicians who take care of patients whose colon cancer might recur should be aware of the relatively high rate of symptomatic recurrences and of typical presenting symptoms.

Ann Fam Med 2016;14:215-220. doi: 10.1370/afm.1919.

\section{INTRODUCTION}

Y early, more than 13,000 patients are diagnosed with colorectal cancer in the Netherlands, and this number is growing due to increasing incidence and aging of the population. ${ }^{1,2}$ The overall 5 -year survival rate of colorectal cancer is approximately $60 \% .{ }^{1}$ After treatment with curative intent, $30 \%$ to $40 \%$ of the patients develop recurrent disease. . $^{3,4}$ Recurrent disease usually presents as distant metastasis in the liver or lungs or as locoregional recurrence in the pelvis or peritoneum. Also the risk of second colorectal malignancy is increased. Most recurrences happen during the first 2 to 3 years after initial treatment. ${ }^{3-5}$

Recurrent disease can be treated with curative intent, depending on the location and number of metastases and on the patient's condition. Early detection of recurrence is therefore important. The Dutch guideline on colorectal cancer includes periodic consultations combined with carcinoembryonic antigen (CEA) testing and imaging of the abdomen during the first 5 postoperative years. Surveillance colonoscopy is recommended 1 year postoperatively, with subsequent colonoscopy every 3 to 5 years depending on the presence, number, size, and location of polyps. ${ }^{2}$

Systematic reviews have shown that intensive follow-up programs with 
frequent diagnostic testing improve overall survival compared with less or no intensive follow-up. ${ }^{3,6}$ Also, the number of attempts at curative reoperations was higher in arms with intensive follow-up.

Tumor-specific guidelines do not offer recommendations about which physician should coordinate follow-up, but recently, primary care physicians were appointed to perform follow-up in the Netherlands. ${ }^{2,7,8}$ Sorting out how recurrent disease presents and is diagnosed during the first 5 postoperative years can help prepare primary care physicians for a future role as coordinators of colon cancer follow-up.

\section{METHODS}

\section{Patients}

In March 2014 all patients operated on for colon cancer between January 2007 and December 2012 in the Academic Medical Centre (AMC) in Amsterdam and the St Antonius Hospital in Utrecht/Nieuwegein were selected from institutional databases. Inclusion criteria were treatment with curative intent for a pathological stage I to stage III carcinoma of the colon, including the rectosigmoid colon, with scheduled follow-up. Patients with stage IV disease, rectal carcinoma, and hereditary colon carcinoma were excluded, as were those whose carcinoma had developed in the context of inflammatory bowel disease and those not eligible for scheduled follow-up. Selection of eligible patients was done by 2 investigators (L.D. and R.H., see acknowledgment) and each randomly checked $50 \%$ of the other's data to ensure reliability.

\section{Follow-Up Schedule}

Follow-up was scheduled according to the Dutch guideline, which includes CEA testing every 3 to 6 months during the first 3 years and every 6 months during the following 2 years. The cutoff point of CEA was $5.5 \mu \mathrm{g} / \mathrm{L}$. Abdominal imaging was performed by ultrasonography every 6 months during the first 2 years and annually during the following 3 years. ${ }^{2}$ At the St Antonius Hospital, imaging of the abdomen was combined with a chest X-ray in all patients, although this was not recommended in the guideline. At the $\mathrm{AMC}$, follow-up during the first 6 months was initiated by the medical oncologist in patients who received adjuvant chemotherapy, after which the surgeon continued follow-up. At the St Antonius Hospital, patients who received adjuvant chemotherapy consulted both surgeons and medical oncologists during 5 years of follow-up, whereas follow-up of patients who only had surgical treatment was performed by surgeons only in both hospitals. In case of elevated CEA level, either another CEA test or further imaging was performed, the latter involving computed tomography (CT) of the thorax and abdomen with or without positron emission tomography (PET).

\section{Characteristics}

We used the hospitals' electronic medical record systems (EMR) to extract the following data: tumor stage and location (ie, right, left, or transverse part of the colon), length of follow-up, and death. In case of recurrent disease, we collected data about the moment recurrence was diagnosed (ie, during a scheduled follow-up visit or not), which physician was involved, the presence of symptoms, the diagnostic tests that raised suspicion of recurrence, and additional diagnostic tests used for definitive diagnosis and further staging. We also captured the location of recurrence, time to diagnosis of recurrence, and survival. Recurrent disease was defined as distant metastasis, locoregional recurrence, or a second colorectal carcinoma confirmed by pathological examination or, if not available, by repeated imaging. Both researchers checked the data of patients with recurrent disease. In case of disagreement, a third researcher (J.W.) was consulted.

\section{Statistical Analysis}

We used descriptive statistical methods to analyze the data by using SPSS v.20.0 package (International Business Machines Corporation). Chi square and Fisher's exact tests were used to compare means. We did a Kaplan-Meier analysis to calculate the overall survival in patients who developed asymptomatic or symptomatic recurrence. The log-rank test was used for comparison. Data of patients who were lost to follow-up after detection of recurrence were censored. The date of the surgical resection of the primary colon cancer was used as starting time for survival analysis.

\section{RESULTS}

During the study period, 823 patients were operated on for colon cancer in the 2 hospitals included in the study. For reasons shown in Table 1, 303 patients were excluded.

Of 520 included patients, 12 ended scheduled follow-up prematurely because of age, 5 requested termination of follow-up, and 4 stopped follow-up because of comorbidity. Eleven patients continued follow-up in hospitals closer to their residences, and 42 were lost to follow-up.

The final analysis included 446 patients, of whom $93(21 \%)$ had stage I carcinoma, 176 (39\%) stage II carcinoma, and 176 (39\%) stage III carcinoma. In 1 patient, stage was unknown. Seven patients had tumor foci at more than 1 site. The majority of patients had 
Eight out of 20 patients with liver metastases were eligible for resection with curative intent. All patients were alive at the end of the analysis, with a median time to last follow-up visit of 20 months (range 1 to 70 months) after liver metastasis detection.

Two patients with recurrent disease were symptomatic during scheduled follow-up visits. Reported symptoms were bowel cramps only and fatigue, diarrhea, and weight loss.

\section{Recurrent Disease Detected Outside Scheduled Follow-up Visits}

Thirty-one of 74 recurrences (42\%) were found during interval visits between scheduled visits. The majority (26/31) of the interval visits were due to symptoms suggestive of recurrent disease. The most prevalent symptoms are shown in Table 4. Five recurrences were asymptomatic and were found during reversal of temporary ostomies $(n=4)$ and during the diagnostic work-up in a patient diagnosed with breast cancer $(n=1)$.

Only 3 patients with recurrent disease detected outside scheduled follow-up visits underwent a surgical treatment with curative intent. Two developed

\section{Table 3. Abnormal Diagnostic Test Results in Patients With Recurrent Disease During Scheduled Follow-Up Visits $(n=43)$}

\begin{tabular}{lr}
\hline Test & No. (\%) \\
\hline CEA tumor marker & $19(44.2)$ \\
Abdominal ultrasound & $17(39.5)$ \\
Colonoscopy & $7(16.3)$ \\
Chest x-ray & $5(11.6)$ \\
CT scan & $3(7)$ \\
More than 1 modality ${ }^{2}$ & $7(16)$ \\
\hline CEA $=$ carcinoembryonic antigen; CT = computed tomography. & \\
a Combinations included CEA testing with abdominal ultrasound $(n=5), C E A$ \\
testing with chest x-ray and abdominal ultrasound $(n=1)$, and CEA testing \\
with CT scan $(n=1)$.
\end{tabular}

Table 4. Symptoms Reported During Interval Visits Leading to Detection of Recurrent Disease $(n=26)$

\begin{tabular}{lc}
\hline Symptom & No. (\%) \\
\hline Abdominal pain & $15(57.7)$ \\
Altered defecation & $11(42.3)$ \\
Weight loss & $6(23.1)$ \\
Pain in back or pelvis & $4(15.4)$ \\
Fatigue & $2(7.7)$ \\
Dyspnea & $2(7.7)$ \\
Loss of appetite & $2(7.7)$ \\
Other & $3(11.6)$ \\
$>1$ symptom & $14(53.8)$ \\
\hline
\end{tabular}

recurrence again after their secondary treatment, and 1 patient with a second primary tumor died. All symptomatic patients with liver metastasis had recurrent disease in multiple locations besides the liver, and none of them could be treated with curative intent.

Surgeons were the physicians most frequently involved if recurrence was suspected during interval visits (14/31). Two symptomatic patients were referred to the hospital by their primary care physicians, and 9 symptomatic patients presented at the emergency department. Other physicians involved were urologists $(\mathrm{n}=3)$, a medical oncologist $(\mathrm{n}=1)$, a gastroenterologist $(\mathrm{n}=1)$, and a cardiologist $(\mathrm{n}=1)$.

The overall survival of patients with recurrent disease differed significantly between the patients with symptomatic and asymptomatic recurrences $(P=.001)$ (Figure 1).

\section{DISCUSSION}

\section{Main Study Findings}

In this retrospective study including 446 colon cancer patients who were treated with curative intent, $17 \%$ developed recurrent disease. Fifty-eight percent of recurrences were detected during scheduled follow-up visits, with $95 \%$ asymptomatic at the time of detection. Another $42 \%$ of the recurrences, however, were discovered outside scheduled follow-up, with $84 \%$ of patients presenting with well-known symptoms such as abdominal pain, altered defecation, and weight loss. Multisite recurrence was detected more often in symptomatic patients. Fifteen patients with asymptomatic recurrence detected during scheduled follow-up were eligible for treatment with curative intent compared with 3 symptomatic patients detected outside scheduled follow-up.

Systematic reviews comparing intensive follow-up with less intensive or no follow-up have found some evidence that intensive follow-up leads to an overall 5 -year survival benefit compared with less intensive or no follow-up. Despite an overall survival benefit, however, the disease-specific survival did not improve significantly because of intensive follow-up. ${ }^{3,6}$ Alteration of lifestyle and improved treatment of coincidental disease were hypothesized to contribute to improved overall survival. ${ }^{9}$ Also, in a recent multicenter trial, patients were randomized to minimal follow-up (ie, follow-up only if symptoms recurred), CEA monitoring only, CT-scanning only, or a combination of CT scanning and CEA monitoring. Compared with minimal follow-up, the absolute difference in the percentage of patients treated with curative intent in the CEA group was $4.4 \%$, in the CT group $5.7 \%$, and in the combined group $4.3 \%$. The number of deaths was 


\section{Figure 1. Overall survival in patients with asymptomatic and symptomatic recurrences.}

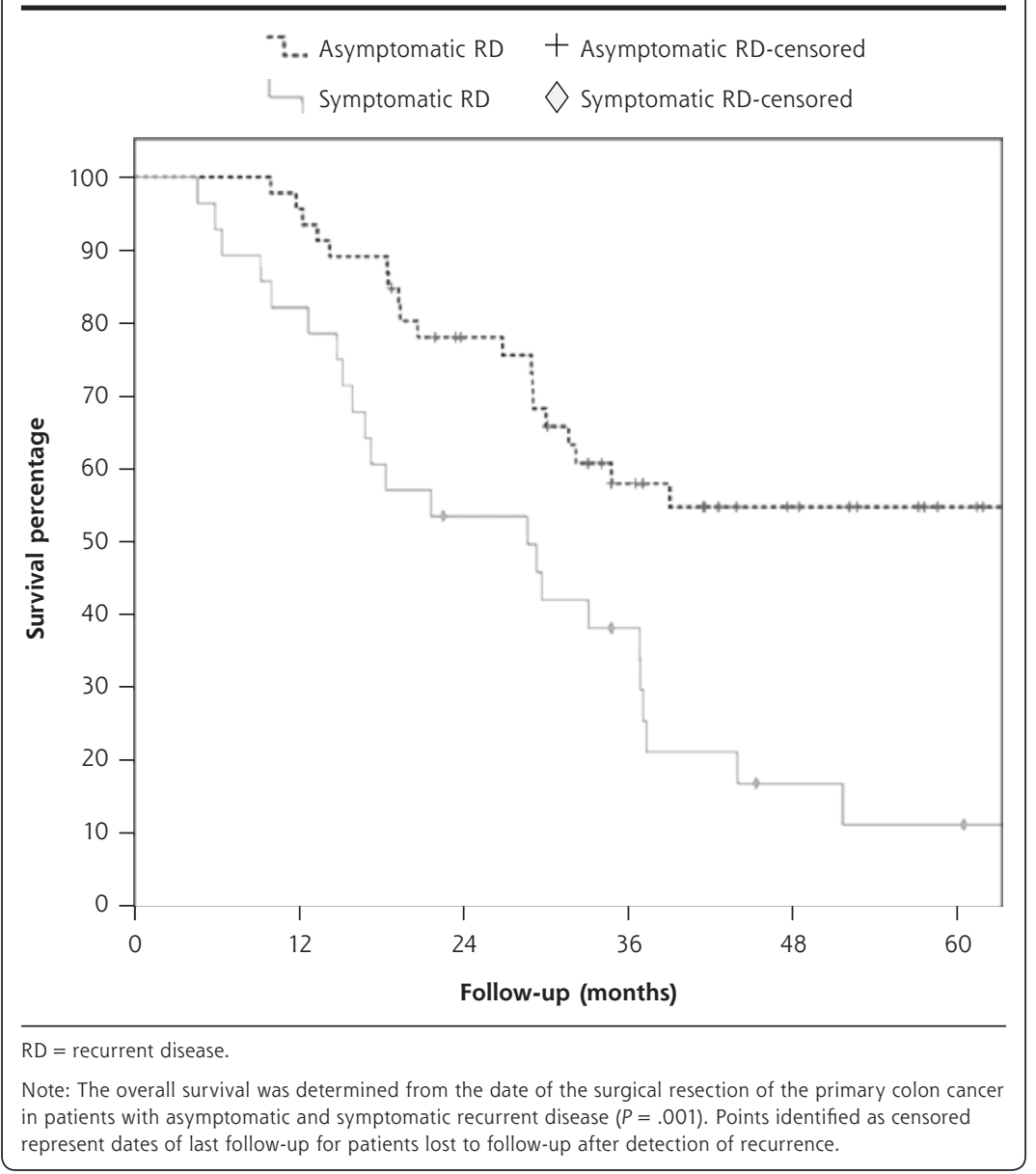

provides a good window of opportunity to detect symptomatic recurrence. ${ }^{14}$ So far, 2 studies have evaluated follow-up and aftercare by primary care physicians, and they found no significant differences for quality of life, recurrence rate, and anxiety. Primary care-led follow-up was more cost-effective than hospital-led follow-up, mainly due to differences in organization and physician costs. ${ }^{15,16}$

\section{Strengths and Limitations}

We were able to extract data of all consecutive patients with a colon carcinoma from 2 hospitals. Given that the quality of the EMR registration of the participating centers depended on involved physicians, it is likely that symptoms of patients with recurrent disease found during scheduled follow-up visits are underreported.

Symptomatic recurrence was mainly registered by surgeons during interval visits. The role of primary care physicians in recognizing the alarming symptoms and referring patients could not be ascertained, since it was not clear how many of the 9 patients presenting to the emergency depart-

not significantly different in the combined intensivemonitoring groups compared with the minimalfollow-up group. ${ }^{10}$

Other aspects of follow-up are important besides timely detection of recurrence- $-\mathrm{eg}$, the patient's quality of life, comorbid diseases, and lifestyle. Patients often experience multiple physical, functional, and psychosocial symptoms after the initial treatment for cancer. These symptoms cause considerable distress. ${ }^{11}$ Care with the purpose of alleviating these symptoms is called aftercare. ${ }^{7}$ Currently, follow-up visits in secondary care do not always address these aspects. Data from the literature suggest that only a small number of distressed patients are identified and supported..$^{12,13}$ Both the Dutch Health Council and the Dutch Cancer Foundation have suggested more generalist care as a solution to these problems. ${ }^{7,8}$ Primary care physicians might be better equipped to guide patients through this difficult and stressful period of their lives. And, since primary health care use is increased during the first 5 years after colorectal cancer diagnosis, this ment with symptoms of recurrence were referred by their primary care physicians.

Scheduled follow-up seems important to detect recurrence, especially during the first 2 years after initial treatment. Forty-two percent of recurrent disease, however, was found during interval visits. Awareness is important when patients present outside scheduled follow-up visits with symptoms of abdominal pain, weight loss, or altered defecation. Considering the fact that primary care-led follow-up might be more costeffective and that more generalist care is desired in the care of patients after their initial treatment, the role of coordinating follow-up care might be shifted toward primary care physicians. To date, little evidence on a more prominent role for them in follow-up of colon cancer patients exists; possible influences on patients' satisfaction and quality of life should be further assessed.

To read or post commentaries in response to this article, see it online at http://www.annfammed.org/content/14/3/215.

Key words: colon cancer; follow-up; recurrent disease 
Submitted May 29, 2015; submitted, revised, November 10, 2015; accepted December 4, 2015.

Prior presentations: These data were presented in part at The Cancer And Primary Care Research International Network meeting; June 10-12, 2014; Winnipeg, Canada, and at The Dutch College of General Practitioners meeting; June, 2014; Groningen, The Netherlands.

Acknowledgments: We thank Robbert B. van den Heuvel for his help in the data collection.

\section{References}

1. Comprehensive Cancer Centre the Netherlands (IKNL). The Netherlands Cancer Registry. https://www.iknl.nl/cijfers/cijfers-over-kanker. Accessed Sep 13, 2014.

2. Comprehensive Cancer Centre the Netherlands (IKNL). Oncoline. National guideline coloncarcinoma. http://www.oncoline.nl/ colorectaalcarcinoom. Published 2014.

3. Jeffery M, Hickey BE, Hider PN. Follow-up strategies for patients treated for non-metastatic colorectal cancer. Cochrane Database Syst Rev. 2007;(1):CD002200.

4. Sargent DJ, Wieand HS, Haller DG, et al. Disease-free survival versus overall survival as a primary end point for adjuvant colon cancer studies: individual patient data from 20,898 patients on 18 randomized trials. J Clin Oncol. 2005;23(34):8664-8670.

5. van Gestel YR, de Hingh IH, van Herk-Sukel MP, et al. Patterns of metachronous metastases after curative treatment of colorectal cancer. Cancer Epidemiol. 2014;38(4):448-454.

6. Tjandra JJ, Chan MK. Follow-up after curative resection of colorectal cancer: a meta-analysis. Dis Colon Rectum. 2007;50(11):1783-1799.
7. Health Council of the Netherlands. Follow-Up in Oncology. Identify Objectives, Substantiate Actions. The Hague, the Netherlands: Health Council of the Netherlands; 2007.

8. Dutch Cancer Society's Signalling Committee on Cancer. Aftercare in Cancer; the Role of Primary Care. Amsterdam, the Netherlands: 2011.

9. Augestad KM, Rose J, Crawshaw B, Cooper G, Delaney C. Do the benefits outweigh the side effects of colorectal cancer surveillance? A systematic review. World J Gastrointest Oncol. 2014;6(5):104-111.

10. Primrose JN, Perera R, Gray A, et al.; FACS Trial Investigators. Effect of 3 to 5 years of scheduled CEA and CT follow-up to detect recurrence of colorectal cancer: the FACS randomized clinical trial. JAMA. 2014;311(3):263-270.

11. Aziz NM. Cancer survivorship research: state of knowledge, challenges and opportunities. Acta Oncol. 2007;46(4):417-432.

12. Berard RM, Boermeester F, Viljoen G. Depressive disorders in an out-patient oncology setting: prevalence, assessment, and management. Psychooncology. 1998;7(2):112-120.

13. Pascoe SW, Neal RD, Allgar VL, Selby PJ, Wright EP. Psychosocial care for cancer patients in primary care? Recognition of opportunities for cancer care. Fam Pract. 2004;21(4):437-442.

14. Heins MJ, Korevaar JC, Rijken PM, Schellevis FG. For which health problems do cancer survivors visit their General Practitioner? Eur J Cancer. 2013:49(1):211-218.

15. Augestad KM, Norum J, Dehof S, et al. Cost-effectiveness and quality of life in surgeon versus general practitioner-organised colon cancer surveillance: a randomised controlled trial. BMJ Open. 2013;3(4).

16. Wattchow DA, Weller DP, Esterman A, et al. General practice vs surgical-based follow-up for patients with colon cancer: randomised controlled trial. Br J Cancer. 2006;94(8):1116-1121. 